\title{
Viral interactions with intermediate filaments: Paths less explored
}

\author{
This article was published in the following Dove Press journal: \\ Cell Health and Cytoskeleton \\ 27 March 2010 \\ Number of times this article has been viewed
}

\section{Samatha Sripada \\ Cecilia Dayaraj}

The Dengue Group, National Institute of Virology, Pune, India
Correspondence: Samatha Sripada Dr. Ambedkar Road, Pune, India 4 I I00 I

$\mathrm{Tel}+912026006240$

Fax +9| 2026122669

Email samatha@august-tech.net National Institute of Virology, 20-A,

\begin{abstract}
The limited repertoire of viruses led to their exploitation of cellular machinery for various events in virus replication, starting from entry till egress. The major cellular contributors in this process are the cytoskeletal components, which serve as entry points into the cells and also as tracks and highways to reach the replication sites. The role of microfilaments and microtubules, two of the major cytoskeletal elements have been extensively studied with respect to virus replication. However, very little is known about the third component, the intermediate filaments. This could in part be due to the intermediate filaments having long been thought to be rigid structures providing mechanical support to the cells. With the increasing knowledge of the dynamic property of intermediate filaments, (owing to their association with microfilaments and microtubules) their role in membrane trafficking and endocytosis, uncovered, the idea that viruses might be exploiting these cytoskeletal elements is gaining impetus. In this review, we have attempted to link viruses with the intermediate filaments, as we believe that intermediate filaments might also be important for virus replication.
\end{abstract}

Keywords: virus replication, microfilaments, microtubules

\section{Introduction}

Intermediate filaments (IF) are the third major type of cytoskeletal elements after microfilaments (MF) and microtubules (MT). They are strong rope like fibers that provide mechanical resilience to the animal cells, which are subject to mechanical stress including epithelial cell, neurons and muscle cells. IF radiate through the cell and are often interconnected to the adjacent cytoskeletal fibers by a family of cross bridging proteins called plectins. ${ }^{1}$ Each plectin molecule contains a binding site for IF at one end and has a binding site for another IF, MF or a MT at the other end. The basic unit of IFs is thought to be a tetramer, formed by two dimers, which become aligned side by side in a staggered fashion with their $\mathrm{N}$ and $\mathrm{C}$ termini diametrically opposed. Because the dimers point in opposite directions, the tetramer itself is nonpolar. Tetramers associate both side-to-side and end-to-end to form intermediates, which in turn form the final filament. ${ }^{2}$

Unlike MF and MT, IF are a chemically heterogeneous group of structures that, in humans, are encoded by more than 60 genes. The polypeptide subunits of IF can be classified into 6 major classes depending on their distribution as well as their biochemical and immunological criteria (Table 1). To this date, the fate of a single IF in a particular cell type has not determined; however, it is certainly evident that they might perform certain specialized functions as shown by their deficiencies in certain cell types. ${ }^{3}$ In the last few years, IFs are gaining greater significance as dynamic elements, performing certain crucial cellular functions rather than being just rigid structures providing mechanical support to the cells. ${ }^{4}$ 
Table I Type and distribution of intermediate filaments in animal cells

\begin{tabular}{llll}
\hline IF protein & Type & Estimated number of polypeptides & Distribution \\
\hline Keratin & I & $>25$ & Epithelia \\
Keratin (basic) & II & $>25$ & Epithelia \\
Vimentin & III & I & Mesenchymal cell \\
Glial Fibrillary Acidic Protein & III & I & Glial cells, astrocytes \\
Peripherin & III & I & Peripheral neurons \\
Lamin proteins & V & I & Nuclear envelopes \\
Nestin & VI & - & Embryonal cells \\
\hline
\end{tabular}

\section{Dynamic nature of IF: Complex interplay of MT and associated motors}

One of the earlier studies by Vikstrom and colleagues showed the dynamic exchange between subunits and polymers of vimentin intermediate filaments at steady state through the use of rhodamine-labeled vimentin in fluorescence recovery after photobleaching (FRAP) analysis. ${ }^{5}$ Recent research undertaken by several groups have shown that bidirectional movements of IFs are dependent on MT and powered by kinesin and dynein. In the absence of IF specific motor proteins, they rely exclusively on actin and MT fibers for their movements. ${ }^{6-8}$ Studies with BHK-21 cells have shown that the assembly of vimentin is a three-step process. Following trypsinization and replating of the cells, vimentin is seen as numerous nonmembrane-bound aggregates, termed particles, throughout the cytoplasm. These aggregates then polymerize to form short vimentin filaments termed squiggles, which in turn form long filaments. At this stage, particles and squiggles are seen only in the peripheral regions of the spreading fibroblasts. Live imaging of cells expressing GFP-vimentin show that filaments constantly assemble, disassemble and exhibit bidirectional saltatory movements along MT tracks. ${ }^{7}$ Confirming the MT based movements; cells injected with anti-kinesin antibodies showed reorganization of IF filaments into perinuclear aggregates. ${ }^{7}$ Similarly kinesin has been shown to be responsible for the movement of other types of IF such as peripherin and neural filaments. ${ }^{7,9}$

Similarly, IF based movements are also shown to depend on the minus end motor protein, dynein complex. Inhibition of dynein function by dynamitin over expression resulted in the reorganization of vimentin and peripherin to the cell surface in BHK-21 and PC12 cells respectively. ${ }^{10}$ Readers are referred to a review by Helfand and colleagues (2004) for a more comprehensive report on dynamics of IF. ${ }^{11}$ Apart from MT based movements, IFs are also known to exhibit actin based movement on myosin Va motor protein. ${ }^{12}$ In cells, all three cytoskeletal elements are closely associated and work in a tightly regulated coordinated fashion. The coordinated functioning of these three components is facilitated by various families of proteins such as plectins, Arp1. Plectins are the proteins that have binding sites for IFs on one side and for MFs or MTs on the other. The interaction of one or more of the three components is often obligatory, especially for intracellular trafficking. In the majority of cases during endocytosis, a sequential participation of MFs and MTs have been proposed where MFs help in the uptake of ligands via endocytosis and MT are involved in the regulation of trafficking between peripheral early endosomes and juxtanuclear late endosomes. ${ }^{13}$

\section{IF in membrane trafficking, vesicular transport and endocytosis}

Recent research has helped elucidate the significance of vimentin IF in: vesicular and organelle transport; regulation of endocytic machinery and organelle positioning; together with assigning a dynamic state for vimentin. ${ }^{14}$ Recent studies have assigned a crucial role for vimentin in the regulation of endocytic and autophagocytic machinery. IFs are known to control AP3 dependent membrane traffic and help in vesicle formation, vesicle budding and vesicle uncoating. IFs may function to recruit MTs or actin based motor to AP3 generated vesicles. ${ }^{14}$

\section{IF in axonal transport and neurodegeneration}

The mammalian nervous system is known to contain eight different IF proteins spread throughout mature neurons and glial cells. Neurofilaments (NFs) and Glial fibriallary acidic protein (GFAP) are predominantly seen in the central nervous system while peripherin is seen in the peripheral nervous system. NFs are synthesized in the neuronal cell body and are transported to axons by virtue of the microtubule transport system. Initially it was thought that the contribution of NFs 
were mostly to the diameter of nerve filaments. However, recent studies with axonal transport indicate that NFs have an important role in neuronal functioning. There have been increasing reports on the correlation of defects in NF trafficking and neurodegeneration. Accumulation of NFs has been shown to cause a variety of neuronal disorders such as: Charcott Marie Tooth Syndrome; Parkinson's Disease; and Amyotrophic Lateral Sclerosis (readers are referred to an excellent review by Perrot and Eyer). ${ }^{15}$ Also, mutant NFs perturb the mitochondrial localization in neurons. Such an event is especially significant because, mitochondrial energy provision is clearly of the utmost importance in neuronal functioning.

\section{IF and virus replication}

The contribution of IFs to virus replication has been an area of investigation since the late 1990s, however; such an association is not as well characterized as in the other two cytoskeletal elements. Several viral infections have been shown to require intact IF networks using acrylamide (ACR) as an IF disruption agent, ${ }^{16}$ but very little is known about the role of the dynamic IFs in viral infections. ${ }^{17,18}$ In this review we summarize the work undertaken by researchers exploring viral interaction with the IF cytoskeleton.

\section{Entry}

Vimentin was shown to participate in the early stages of HIV 1 replication, by aiding the uptake of HIV 1 DNA into the nuclear compartment. Inhibition of vimentin with specific antisera showed a reduction in the amount of HIV 1 Gag specific RNA in the nuclei of monocytes, following infection with HIV 1 pseudovirions. Vimentin binds to the third hypervariable region (V3 loop) of the envelope protein gp120 and subsequently induces the viral entry and translocation of viral DNA. ${ }^{19}$ The authors here suggest that vimentin could be used as a receptor during virion internalization. Confocal microscopy and inhibitor assays showed a close association between Vif and vimentin; indeed the intracellular localization of Vif is dependent on the presence of the intermediate filament vimentin. ${ }^{20}$ The association of Vif with vimentin can cause the collapse of the intermediate filament network into a perinuclear aggregate; similarly, analysis of Vif in vimentin-negative cells reveals significant staining of the nucleus and the nuclear membrane, in addition to diffuse cytoplasmic staining. The interaction of Vif with vimentin according to the authors is necessary for the regulation of virus maturation or for virus entry into a host cell possibly via vimentin network.
Porcine respiratory and reproductive syndrome virus (PRRSV) binds to and modulates the expression of cell surface vimentin and is endocytosed into MARC cells. ${ }^{21}$

Chen et al, ${ }^{22,23}$ showed that dengue virus (DENV) infection of ECV304 cells required vimentin. Confocal analysis showed DENV infection induced MT and vimentin reorganization in ECV304 cells. Acrylamide treatment inhibited virus infection suggesting that vimentin is important for virus replication. Studies in our own laboratory have shown that DENV infection requires an intact IF network at all stages of virus replication. However, it is especially important during virus uncoating. Significant reorganization of vimentin filaments to form a vimentin cage has been observed during virus assembly (Figure 1).

\section{IF and virus assembly}

IFs are known components of specialized structures called aggresomes. Aggresomes are sites of misfolded protein accumulation, which are eventually degraded by host cell proteases. Ultrastructural examination of aggresomes indicates a complex architecture of individual particles dispersed with subcellular organelles and filaments surrounded by membranous organelles and vimentin fibers. The function of IFs is to form a protective cage around the aggresomes preventing the release of the toxins into the cytosol. ${ }^{23}$

Assembly of large DNA viruses such as African swine fever virus (ASFV) and herpes virus was shown to occur in discrete perinuclear foci called viral factories. Recent studies have shown that these viruses hijack the aggresome pathway for assembly. ${ }^{24,25}$ These viral factories are located close to the microtubule-organizing center (MTOC) and require an intact microtubule network for assembly. These are characterized by the rearrangement of IFs and the collapse of vimentin into characteristic cages around the viral factories. It is speculated that viruses use aggresome pathways to concentrate cellular and viral proteins that facilitate replication and assembly. It is suggested that vimentin may provide a mechanical scaffold for the recruitment of viral proteins necessary for virus genome replication and assembly. For example, ASFV DNA replication results in phosphorylation of vimentin by calcium calmodulin-dependent protein kinase II (CaM kinase II) and the concomitant conversion of vimentin aster into a cage. Initial rearrangement of vimentin into an aster at the MTOC was believed to be required for the formation of the virus assembly site, perhaps by providing a scaffold for the recruitment of viral proteins necessary for virus DNA replication. Soon after the onset of viral DNA replication, vimentin is rearranged into a cage around the 


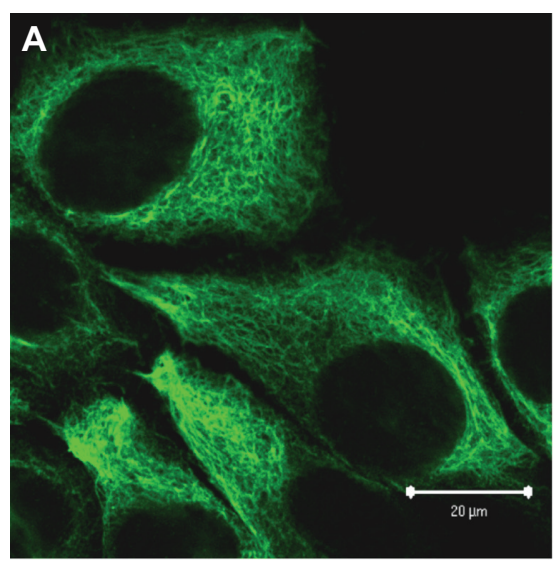

Vimentin

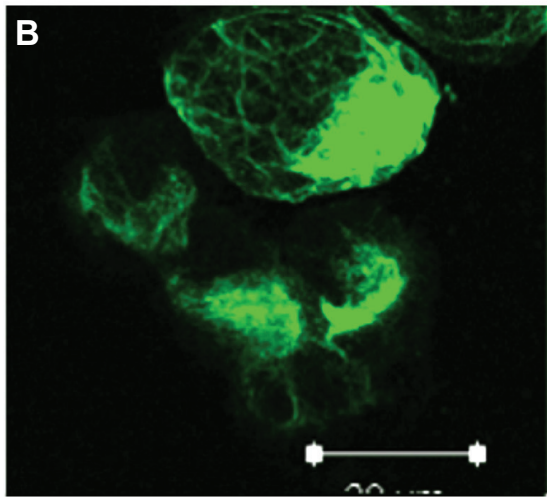

Dengue Virus

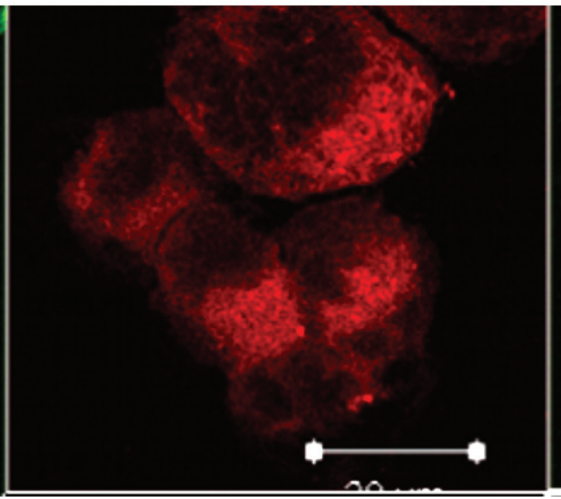

Merge

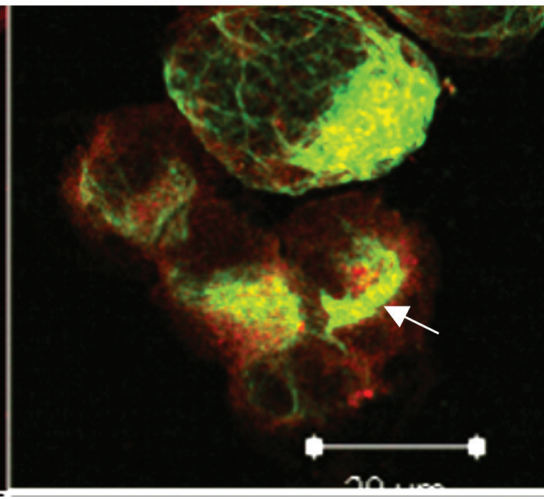

Figure I Association of DENV with vimentin filaments during replication. PS cells infected with DENV were fixed at $24 \mathrm{~h}$ p.i. and labeled for vimentin (green) and virus (red). A) Mock infected PS cell showing a radiating IF structure. B) Split image of DENV-2 infected cells showing vimentin cage indicated by arrow. Bars $20 \mu$ M.

factory. Here, the functions of the vimentin cage might be the prevention of movement of viral components into the cytoplasm. ${ }^{26}$ The study also showed that the rearrangement of vimentin was dependent on MTs, since vimentin cages were lost in cells treated with nocodazole or expressing p50/dynamitin. Serine phosphorylation of vimentin by CaM II kinase is thought to reduce interactions between individual vimentin filaments and facilitate the redistribution of intermediate filaments into a vimentin cage thereby accommodating viral genome and the virus factory.

Risco and colleagues showed that elements of endoplasmic reticulum golgi intermediate compartment (ERGIC) and vimentin filaments concentrate in the viral factories of vaccinia virus (VV). Their results showed that VV p39 core protein colocalizes with vimentin filaments that have been recruited in the perinuclear viroplasm foci. Based on their results they proposed that modified cellular ERGIC membranes and vimentin intermediate filaments act coordinately in the construction of viral factories and the first VV form through a unique mechanism of viral morphogenesis from cellular elements. They also suggest that in addition to building a protective cage, modified vimentin could play a more dynamic role, such as organizing the interior of viroplasm foci or facilitating egress and incorporation of viral proteins inside immature viral particles. ${ }^{27,28}$

A recent study by Nitahara-Kasahara and colleagues ${ }^{28,29}$ suggested that modulation of hepatic vimentin expression might enable the control of hepatitis $\mathrm{C}$ virus (HCV) production. Comparative proteomic studies of proteins in HCV core-expressing and non-expressing Huh7 cell lines, Uc39-6 and Uc321, respectively, showed that coreexpressing Uc39-6 cells had much lower vimentin content than Uc321 cells. Similarly, vimentin-overexpressing and vimentin-knocked-down cells, demonstrated varying levels of cellular vimentin content. While underexpression of vimentin in vimentin null cells had no effect on mRNA level of core protein and proteasome-dependent degradation of the core protein was strongly reduced, indicating that the turnover rate of core protein is regulated by cellular vimentin content. 


\section{Virus egress}

The degradation of vimentin in the late stages of viral infection is known for several animal viruses..$^{29-33}$ The full importance of these alterations is not yet understood, however it is argued by Doorbar and colleagues that degradation of the cytokeratin matrix removes the physical barrier, thus facilitating viral release. Late in infection, IFs are cleaved by several viruses and such degradation of the cytoskeletal network was shown to be necessary for the release of nascent virions. ${ }^{30,34}$ Brown and colleagues showed that actin is used as a cofactor in the degradation of the cytokeratin network by adenoviral proteinase. ${ }^{35}$ Although it is known that degradation of cytoskeletal elements helps in release of nascent virions, it is unusual that a cellular protein is used as a cofactor in the degradation of the cytokeratin network.

Vimentin was shown to be necessary for the trafficking of blue tongue virus to the cell surface. Pharmacological disruption of the vimentin network leads to an accumulation of the cell associated virus and a decreased release of blue tongue virus. ${ }^{36}$

\section{Neural transmission of viruses}

The involvement of IFs has been shown in the neural transmission of avian influenza virus (AIV) and pseudorabies virus (PRV). ${ }^{37}$ Treatment with cytoskeletal inhibitors showed significant suppression of the spread of AIV and PRV in the acrylamide treated cultured neurons. The authors suggest that intact IFs are involved in the transmission of the viruses in the neuronal network, ie, the neural spread of virus. Intact IF structure was also shown to be important for replication of human cytomegalovirus (CMV). Miller and Hertel showed that disruption of the vimentin network with acrylamide in IF bundling in cells from a patient with giant axonal neuropathy, and the absence of vimentin in fibroblasts from vimentin null mice, greatly reduced entry of two genotypically distinct strains of CMV, one with narrow (AD169) and one with broad (TB40/E) cell tropism. In vimentin null cells, viral particles remained in the cytoplasm longer than in vimentin $(+/+)$ cells. These findings demonstrate that an intact vimentin network is required for viral entry to facilitate capsid trafficking and/or docking to the nuclear envelope. ${ }^{38}$

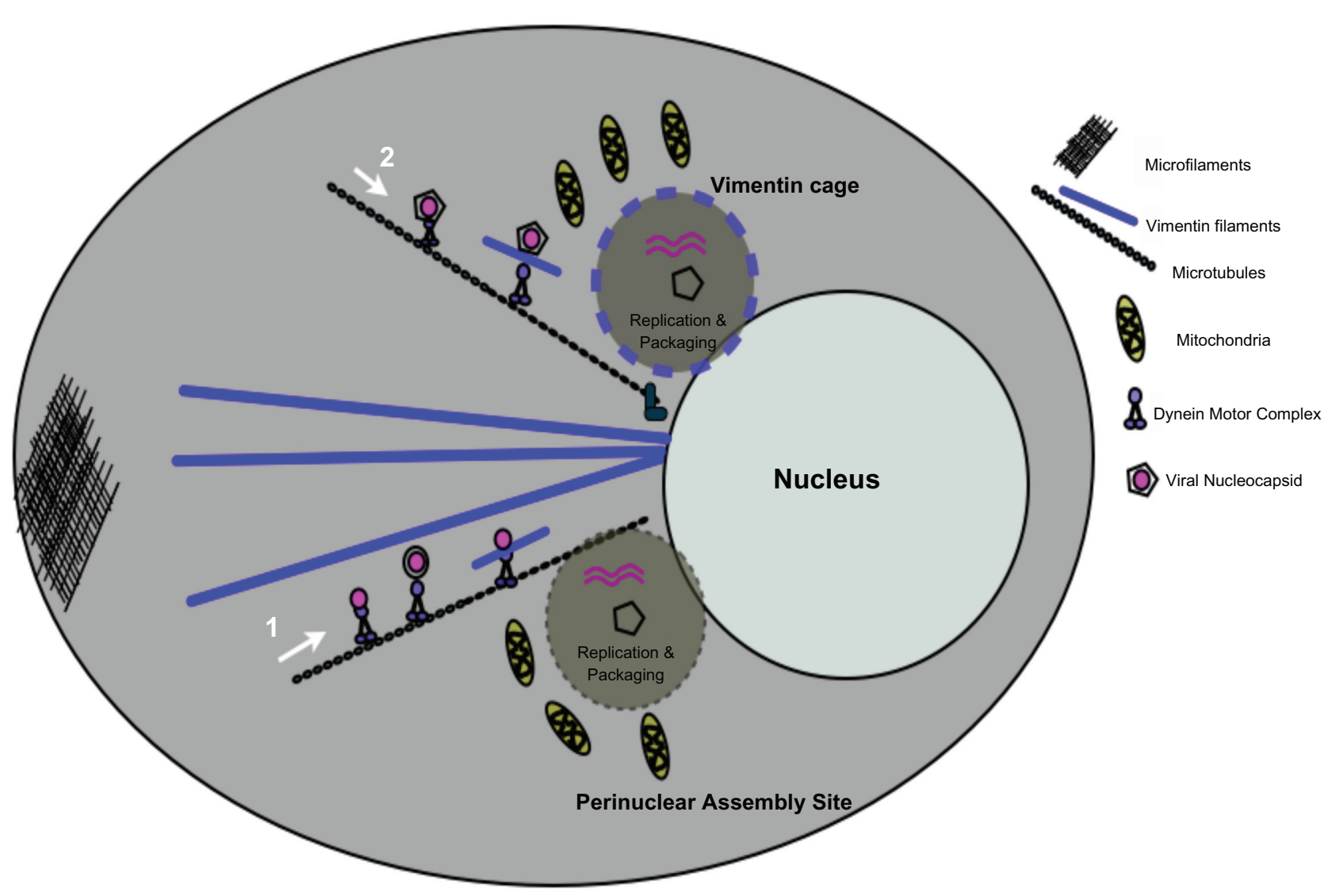

Figure 2 Virus assembly and Intermediate filaments.

Route I: Viral nucleocapsids are transported along the microtubules via the dynein complex directly or via vimentin squiggles to the perinuclear assembly region comprising of endoplasmic reticulum and Golgi apparatus.

Route 2: Viruses such as Poxvirus and African Swine fever virus deliver their cores enter the cytoplasm which are transported to perinuclear virus factory that induces vimentin rearrangement and recruitment of mitochondria and chaperones to factory precursors, similar to an aggresome. 


\section{Conclusions and future perspective}

It is now apparent that IF have more to contribute to virus replication than has been originally understood. They have been principally shown to contribute during virus entry and also during virus assembly where they are supposed to provide mechanical support to ongoing virus replication (Figure 2). Further studies however are required to understand the significance of IFs in virus replication. Questions that still need to be addressed:

1. Do viral interactions with IFs have more to contribute to pathogenicity especially in the case of neurotropic viruses?

2. Does any kind of regulatory switch dictate viral interactions with IFs during the entry and egress of viruses?

3. Does virus manipulation of IFs arise as a prerequisite of the assembly or replication strategy, as in case of aggresomes?

4. Does virus interaction with IFs during entry have significant implications in endocytosis?

\section{Acknowledgements}

We acknowledge Dr AC Mishra, Director, National Institute of Virology, Pune, India and Indian Council of Medical Research, New Delhi, India for their support. Sripada is supported by Graduate Student Fellowship from Council of Scientific and Industrial Research.

\section{Disclosures}

The authors report no conflict of interest relevant to this research.

\section{References}

1. Svitkina TM, Verkhovsky AB, Borisy GG. Plectin sidearms mediate interaction of intermediate filaments with microtubules and other components of the cytoskeleton. J Cell Biol. 1996;135(4):991-1007.

2. Lodish H, Matsudaira P, Berk A, Ploegh H, Scott MP. Microfilaments and Intermediate filaments. In: Steyen $\mathrm{R}$ and DiVittorio S, editors. Molecular Biology of Cell. New York, NY: W.H. Freeman and Co; 2004. p. 795-891.

3. Omary MB. "IF-pathies": a broad spectrum of intermediate filamentassociated diseases. J Clin Invest. 2009;119(7):1756-1756.

4. Styers ML, Kowalczyk AP, Faundez V. Intermediate filaments and vesicular membrane traffic: The odd couple's first dance? Traffic. 2005;6(50):359-365.

5. Vikstrom KL, Lim SS, Goldman RD, Borisy GG. Steady state dynamics of intermediate filament networks. J Cell Biol. 1992;118(1):121-129.

6. Prahlad V, Yoon M, Moir RD, Vale RD, Goldman RD. Rapid movements of vimentin on microtubule tracks: kinesin-dependent assembly of intermediate filament networks. J Cell Biol. 1998;43(1):159-170.

7. Yoon M, Moir RD, Prahlad V, Goldman RD. Motile properties of vimentin intermediate filament networks in living cells. $J$ Cell Biol. 1998;143(1):147-157.

8. Helfand BT, Mikami A, Vallee RB, Goldman RD. A requirement for cytoplasmic dynein and dynactin in intermediate filament network assembly and organization. J Cell Biol. 2002;157(5):795-806.
9. Helfand BT, Chang L, Goldman RD. The dynamic and motile properties of intermediate filaments. Annu Rev Cell Dev Biol. 2003;19:445-467.

10. Helfand BT, Loomis P, Yoon M, Goldman RD. Rapid transport of neural intermediate filament protein. $J$ Cell Sci. 2003;116(11):2345-2359.

11. Helfand BT, Chang L, Goldman RD. Intermediate filaments are dynamic and motile elements of cellular architecture. J Cell Sci. 2004;117(2):133-141.

12. Rao MV, Engle LJ, Mohan PS, et al. Myosin Va binding to neurofilaments is essential for correct myosin Va distribution and transport and neurofilament density. J Cell Biol. 2002;159(2):279-290.

13. Durrbach A, Louvard D, Coudrier E. Actin filaments facilitate two steps of endocytosis. J Cell Sci. 1996;109(2):457-465.

14. Styers ML, Salazar G, Love R, Peden AA, Kowalczyk AP, Faundez V. The endo-lysosomal sorting machinery interacts with the intermediate filament cytoskeleton. Mol Biol Cell. 2004;15(12):5369-5382.

15. Perrot R, Eyer J. Neuronal intermediate filaments and neurodegenerative disorders. Brain Res Bull. 2009;80(4-5):282-295.

16. Bukrinskaya A, Brichacek B, Mann A, Stevenson M. Establishment of functional human immunodeficiency virus type 1 (HIV1) reverse transcription complex involves the cytoskeleton. J Exp Med. 1998; 188:2113-2125.

17. Eckert BS. Alte+ration of intermediate filament distribution in PtK1 cells by acrylamide. Eur J Cell Biol. 1985;37:169-174.

18. Arcangeletti MC, Pinardi F, Missorini S, et al. Modification of cytoskeleton and prosome networks in relation to protein synthesis in influenza A virus-infected LLC-MK2 cells. Virus Res. 1997;51(1):19-34.

19. Cordo SM, Candurra NA. Intermediate filament integrity is required for Junin virus replication. Virus Res. 2003;97(1):47-55.

20. Thomas EK, Connelly RJ, Pennathur S, Dubrovsky L, Haffar OK, Bukrinsky MI. Anti-idiotypic antibody to the V3 domain of gp120 binds to vimentin: a possible role of intermediate filaments in the early steps of HIV-1 infection cycle. Viral Immunol. 1996;9(2):73-87.

21. Karczewski MK, Strebel K.Cytoskeleton association and virion incorporation of the human immunodeficiency virus type 1 Vif protein. J Virol. 1996;70(1):494-507.

22. Kim JK, Fahad AM, Shanmukhappa K, Kapil S. Defining the cellular target(s) of porcine reproductive and respiratory syndrome virus blocking monoclonal antibody 7G10. J Virol. 2006;80(2):689-696.

23. Chen W, Gao N, Wang JL, Tian YP, Chen ZT, An J. Vimentin is required for dengue virus serotype 2 infection but microtubules are not necessary for this process. Arch Virol. 2008;153(9):1777-1781.

24. Johnston JA, Ward CL, Kopito RR. Aggresomes: a cellular response to misfolded proteins. J Cell Biol. 1998;143(7):1883-1898.

25. Heath CM, Windsor M, Wileman T. Aggresomes resemble sites specialized for virus assembly. $J$ Cell Biol. 2001;153(3):449-455.

26. Nozawa N, Yamauchi Y, Ohtsuka K, Kawaguchi Y, Nishiyama Y. Formation of aggresome-like structures in herpes simplex virus type 2-infected cells and a potential role in virus assembly. Exp Cell Res. 2004;299(2):486-497.

27. Stefanovic S, Windsor M, Nagata KI, Inagaki M, Wileman T. Vimentin rearrangement during African swine fever virus infection involves retrograde transport along microtubules and phosphorylation of vimentin by calcium calmodulin kinase II. J Virol. 2005;79(18):11766-11775.

28. Risco C, Rodríguez JR, López-Iglesias C, Carrascosa JL, Esteban M, Rodríguez D. Endoplasmic reticulum-Golgi intermediate compartment membranes and vimentin filaments participate in vaccinia virus assembly. J Virol. 2002;76(4):1839-1855.

29. Nitahara-Kasahara Y, Fukasawa M, Shinkai-Ouchi F, et al. Cellular vimentin content regulates the protein level of hepatitis $\mathrm{C}$ virus core protein and the hepatitis $\mathrm{C}$ virus production in cultured cells. Virology. 2009;383(2):319-327.

30. Doorbar J, Ely S, Sterling J, McLean C, Crawford L. Specific interaction between HPV-16 E1-E4 and cytokeratins results in collapse of the epithelial cell intermediate filament network. Nature. 1991;352:824-827.

31. Leão Ferreira RL, Moussatche N, Neto VM. Rearrangement of intermediate filament network of BHK-21 cells infected with vaccinia virus. Arch Virol. 1994;138:273-285. 
32. Murti KG, Goorha R, Klymkowsky MW. A functional role for intermediate filaments in the formation of frog virus 3 assembly sites. Virology. 1988;162(1):264-269.

33. Karczewski MK, Strebel K. Cytoskeleton association and virion incorporation of the human immunodeficiency virus type 1 Vif protein. J Virol. 1996;70(1):494-507.

34. Nédellec P, Vicart P, Laurent-Winter C, Martinat C, Prévost MC, Brahic M. Interaction of Theiler's virus with intermediate filaments of infected cells. J Virol. 1998;72(12):9553-9560.

35. Brown MT, McBride KM, Baniecki ML, Reich NC, Marriott G, Mangel WF. Actin can act as a cofactor for a viral proteinase in the cleavage of the cytoskeleton. J Biol Chem. 2002;277(48): 46298-46303.
36. Bhattacharya B, Noad RJ, Roy P. Interaction between Bluetongue virus outer capsid protein VP2 and vimentin is necessary for virus egress. Virol J. 2007;4:7.

37. Matsuda K, Shibata T, Sakoda Y, et al. In vitro demonstration of neural transmission of avian influenza A virus. J Gen Virol. 2005;86(4) 1131-1139.

38. Miller MS, Hertel L. Onset of human cytomegalovirus replication in fibroblasts requires the presence of an intact vimentin cytoskeleton. J Virol. 2009;83(14):7015-7028.

\section{Publish your work in this journal}

Cell Health and Cytoskeleton is an international, peer-reviewed open access journal focusing on all aspects of cell structure and function contributing to normal physiology and cell health and exploring the pathogenesis of cell dysfunction leading to adverse conditions and disease in the organism. The journal welcomes papers covering original research,

\section{Dovepress}

basic science, reviews and evaluations, guidelines, expert opinion and commentary, case reports and extended reports. The manuscript management system is completely online and includes a very quick and fair peerreview system, which is all easy to use. Visit http://www.dovepress.com/ testimonials.php to read real quotes from published authors.

Submit your manuscript here: http://www.dovepress.com/cell-health-and-cytoskeleton-journal 